\title{
Spinor Bosonic Atoms in Optical Lattices: Symmetry Breaking and Fractionalization
}

\section{Citation}

Demler, Eugene, and Fei Zhou. 2002. "Spinor Bosonic Atoms in Optical Lattices: Symmetry Breaking and Fractionalization." Physical Review Letters 88 (16) (April 3). doi:10.1103/ physrevlett.88.163001.

\section{Published Version}

doi:10.1103/PhysRevLett.88.163001

\section{Permanent link}

http://nrs.harvard.edu/urn-3:HUL.InstRepos:27988568

\section{Terms of Use}

This article was downloaded from Harvard University's DASH repository, and is made available under the terms and conditions applicable to Other Posted Material, as set forth at http:// nrs.harvard.edu/urn-3:HUL.InstRepos:dash.current.terms-of-use\#LAA

\section{Share Your Story}

The Harvard community has made this article openly available.

Please share how this access benefits you. Submit a story.

\section{Accessibility}




\title{
Spinor Bosonic Atoms in Optical Lattices: Symmetry Breaking and Fractionalization
}

\author{
Eugene Demler ${ }^{1}$ and Fei Zhou ${ }^{2, *}$ \\ ${ }^{1}$ Physics Department, Harvard University, Cambridge, Massachusetts 02138 \\ ${ }^{2}$ NEC Institute, 4 independence way, Princeton, New Jersey 08540
}

(Received 10 May 2001; published 3 April 2002)

\begin{abstract}
We study superfluid and Mott insulator phases of cold spin-1 Bose atoms with antiferromagnetic interactions in an optical lattice, including a usual polar condensate phase, a condensate of singlet pairs, a crystal spin nematic phase, and a spin singlet crystal phase. We suggest a possibility of exotic fractionalized phases of spinor Bose-Einstein condensates and discuss them in the language of $Z_{2}$ lattice gauge theory.
\end{abstract}

DOI: $10.1103 /$ PhysRevLett.88.163001

Recent experiments on the condensation of Bose gases with internal degrees of freedom initiated a considerable amount of work on understanding the nature of spinor Bose-Einstein condensates (BEC). In particular Bose condensation in alkali atoms, with nuclear spin $I=3 / 2$ that have three low energy hyperfine states and therefore behave as hyperfine spin $F=1$ bosons, has been the subject of active experimental [1-4] and theoretical research [5-10]. It was pointed out that the ground state of atoms with antiferromagnetic interactions in an optical trap is not a usual condensate of a macroscopic number of atoms in a single quantum state, but, rather, a complicated many body state of atoms arranged into a total singlet [7-10]. In this article we examine the problem of Bose $F=1$ atoms with antiferromagnetic interaction (spin symmetric interactions are assumed throughout the paper) in optical lattices arrays of microscopic potentials created by interfering laser beams [11-18]. The dynamics of spinless bosonic atoms in arrays of optical wells may be described by a BoseHubbard model with the possibility of quantum phase transitions between insulating and superfluid phases induced by varying the properties of the laser light [19]. This transition has recently been observed in experiments [20,21]. Here, we propose several new phases that will appear for $F=1$ bosons due to an interplay between spin and charge degrees of freedom: singlet pair condensate that only breaks charge symmetry, a spin nematic crystal phase that only breaks spin symmetry, and a novel "strong coupling pairing (SCP) phase" that breaks both spin and charge symmetry but is distinct from a simple polar BEC. These phases may be considered as "fragmented condensates" [22], and have fractionalized topological excitations: halfvortices and $\pi$ disclinations. Josephson-type experiments may be used to distinguish among insulating and various superfluid phases. We also conjecture the possibility of fractionalized phases in spinor Bose gases. Fractionalization is characterized by a topological order [23-25] and may coexist with any broken continuous symmetry [26].

In what follows we consider the case of antiferromagnetic interaction between the atoms $a_{0}>a_{2}$, where $a_{F}$ is an $s$-wave scattering length in the $F$ channel. This is the case, for example, for ${ }^{23} \mathrm{Na}$ atoms [5]. In a single
PACS numbers: 32.80.Pj, 03.75.Fi, 71.35.Lk

well a faithful representation of the possible spin states of $F=1$ atoms is given by the spherical wave functions $Y_{L m}(\mathbf{n})$, where $\mathbf{n}$ is a unit vector (we assume that all the atoms are in the same orbital state, which is the ground state for the confining potential). The total (collective) spin of the atoms and its $z$ projection correspond to $L$ and $m$, respectively. The symmetry of the bosonic wave function is enforced by the constraint $L+N=$ even, and condition $L \leq N$ gives the correct Hilbert space. Hence, we can write the spin state of the bosons as a wave function on a unit sphere [10] $|\psi\rangle_{N}=\int_{\mathbf{n}} \psi(\mathbf{n})|N, \mathbf{n}\rangle$, provided that the angular momemtum $\mathbf{L}=-i \mathbf{n} \times \frac{\partial}{\partial \mathbf{n}}$ for the wave function $\psi(\mathbf{n})$ satisfies the constraints discussed above. When the number of particles $N$ is large one can give a simple interpretation of the wave functions $|N, \mathbf{n}\rangle$ as $\frac{1}{\mathcal{N}}\left(n_{x} a_{x}^{\dagger}+n_{y} a_{y}^{\dagger}+n_{z} a_{z}^{\dagger}\right)^{N}|0\rangle$ [9]. Here $a_{\alpha}^{\dagger}$ are boson creation operators in the representation where they transform as vectors under spin rotations, $|0\rangle$ is the vacuum state, and $\mathcal{N}$ is a normalization factor.

The Hamiltonian of an array of identical optical wells with spin symmetric tunneling is given by [10]

$$
\begin{aligned}
\mathcal{H} & =\sum_{i} \mathcal{H}_{i}+\sum_{i j} \mathcal{H}_{i j}, \\
\mathcal{H}_{i} & =\frac{u}{2} N_{i}^{2}-\mu N_{i}+\frac{g}{2} \mathbf{L}_{i}^{2}, \\
\mathcal{H}_{i j} & =-2 t \mathbf{n}_{i} \mathbf{n}_{j}\left(b_{i}^{\dagger} b_{j}+b_{j}^{\dagger} b_{i}\right),
\end{aligned}
$$

where we defined the charge creation and annihilation operators that change the number of particles $N_{i}$, but not the direction of $\mathbf{n}_{i}: \quad b_{i}^{\dagger}\left|N_{i}, \mathbf{n}_{i}\right\rangle=\left(N_{i}+1\right)^{1 / 2}\left|N_{i}+1, \mathbf{n}_{i}\right\rangle$, the number of particles in each well $N_{i}=b_{i}^{\dagger} b_{i}$, and the angular momentum operators $\mathbf{L}_{i}=-i \mathbf{n}_{i} \times \frac{\partial}{\partial \mathbf{n}_{i}}$ that describe a collective spin in well $i[7,10]$. Constraints $N_{i}+L_{i}=$ even and $L_{i} \leq N_{i}$ are implied for all $i$, and for simplicity in Eq. (1) we chose to work in the grand canonical ensemble using a chemical potential $\mu$. For a single well Hamiltonian (1) correctly gives a spin singlet ground state if the number of particles is even [7-10]. When optical lattices are produced by lasers with wavelength $\lambda$, they create an optical potential $V(\mathbf{x})=V_{0} \sum_{i} \sin ^{2}\left(k x_{i}\right)$. We can estimate the parameters 
of the Hamiltonian (1) as $g=\frac{2 \pi^{2}}{3} E_{R} \frac{\left(a_{0}-a_{2}\right)}{\lambda} \zeta^{3}$, $U=\frac{2 \pi^{2}}{3} E_{R} \frac{\left(a_{0}+2 a_{2}\right)}{\lambda} \zeta^{3}, t=\frac{2}{\pi^{1 / 2}} E_{R} \zeta^{3} e^{-2 \zeta^{2}}$, where $E_{R}=$ $\hbar^{2} k^{2} / 2 M$ is the recoil energy and $\zeta=\left(\frac{V_{0}}{E_{R}}\right)^{1 / 4}$. The obvious parameters to tune in experiments are the number of particles per well (e.g., by varying boson density) and the strength of periodic potential $V_{0}$ (by tuning the laser intensity). When the latter is varied it affects tunneling exponentially and the interaction coefficients only as a power law. In what follows, we therefore take $U$ and $g$ as constant and consider a phase diagram in the coordinates $t$ and $\mu$.

There are two limits when construction of the phase diagram of Eq. (1) is simple. When $g=0$ there is no energetic penalty for having high angular momentum (nonzero spin) states of quantum rotors in Eq. (1) and spin symmetry is broken. For integer filling factors we have spin nematic insulator (NI) phases for small $t$ and a superfluid polar condensate (PC) phase for larger values of $t$. For fractional filling factors the system is always superfluid. The other simple case is when $g$ is large, and states with odd number of atoms in individual wells are energetically costly: they are not allowed to have an $L=0$ state of a rotor due to parity constraint and they have a higher rotational energy. It is energetically favorable to have even numbers of atoms in each well arranged into singlet combinations, which can be interpreted as binding of atoms into singlet pairs with a pair binding energy $g$. In the limit when $g>U$ the crystal phases are possible for even numbers of atoms per well only and correspond to spin singlet insulators (SSI). The superfluid phase is a condensate of singlet pairs, in which tunneling of individual atoms between the wells is suppressed and only singlet pairs are delocalized. The origin of pairing in this case is a singlet formation on the scale of individual wells. This is reminiscent of the "attraction from repulsion" mechanism of electron pairing proposed by Chakravarty and Kivelson for high $T_{c}$ cuprates, $C_{60}$, and polyacenes [27]. In the case of $0<g<U$ we expect Mott crystal phases for all integer filling factors. For even $N$ 's and small $t$ the rotors' kinetic energy dominates and we have singlet insulator phases (SSI). When $t$ is increased the system goes to a spin NI phase that has an admixture of $L \neq 0$ in the ground state. For odd $N$ 's when all charge fluctuations are frozen there is always at least $L=1$ on each site, so we expect the system to be analogous to the spin-1 lattice model with a broken spin symmetry. The superfluid phase is a PC for large $t$ but may be a spin singlet fluid of pairs for small $t$. There may also exist other phases that break translational symmetry, but they will be discussed in future publications [28].

The scale for the energy difference (per well) between various phases discussed above is set by $g$. By taking parameters similar to the ones used in the experiments of Greiner et al. [21] we estimate $g$ to be of the order of tens of hertz. Hence, we expect that the phases proposed above may be observed with the current experimental techniques. Josephson-type oscillations of the current may be used to distinguish the two superfluid phases. We imag- ine spinor atoms in an optical lattice in the presence of gravity. Gravity acts as voltage in conventional Josephson junction arrays and gives rise to oscillations in the current [20]. Josephson oscillations may be measured selectively in spin or may measure all the particles regardless of their spin. In the case of a single atom condensate (PC) we expect oscillations for both kinds of measurements with frequent $\hbar \omega_{J}=m g \lambda / 2$. A singlet pair condensate will only give oscillations in the second channel with frequency $2 \hbar \omega_{J}=m g \lambda$. Insulating phases will not show any Josephson oscillations, and nematic vs spin singlet may be distinguished further through different correlations for particles of different spin.

Order parameters for the various phases suggested above and shown in Fig. 1 may be conveniently discussed using a Euclidean space-time action. We implement the constraint $N_{i}+L_{i}=$ even using a projection operator $P_{i}=\frac{1}{2} \sum_{\sigma_{i}= \pm 1} e^{i(\pi / 2)\left(1-\sigma_{i}\right)\left(N_{i}+L_{i}\right)} \quad$ [26]. For simplicity we assume that the average number of particles per well is large, and constraint $L_{i} \leq N_{i}$ may be neglected (the qualitative picture derived from its analysis should, however, apply even when this number is of the order of 1). To calculate the partition function $Z=\operatorname{Tr}\left[e^{-\beta \mathcal{H}} P\right]$ we divide imaginary time $\beta$ into $M$ slices of length $\epsilon=\beta / M$ and, after a few standard manipulations that include the

(a)

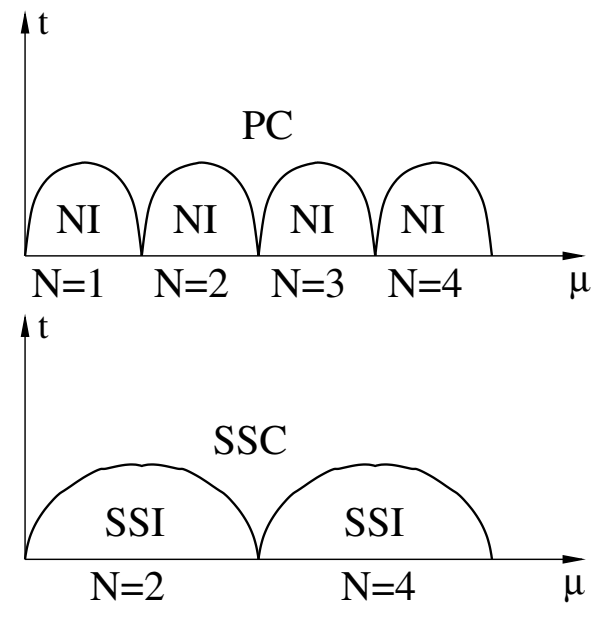

(b)

(c)

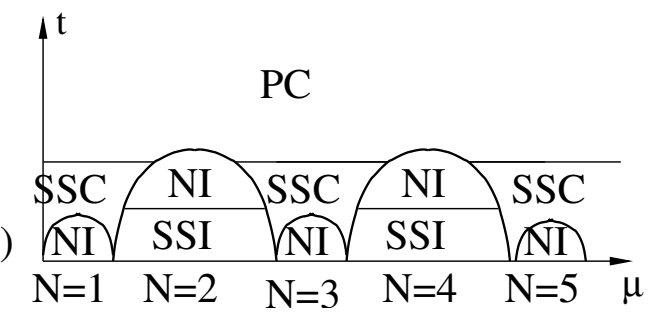

FIG. 1. Phase diagram of the Hamiltonian (1). (a) This corresponds to $g=0$, (b) corresponds to $g>U$, and (c) corresponds to the case $0<g<U$. PC is the polar condensate phase, NI is the nematic insulator, SSC is the spin singlet condensate, and SSI is the spin singlet insulator. The topology of the phase diagram has not been studied in detail. For example, it is possible that in (c) there is a direct phase transition between phases PC and SSI. 
Hubbard-Stratanovich transformation of the last term in Eq. (1), we find

$$
\begin{aligned}
S= & -\sum_{r r^{\prime}} J_{r r^{\prime}}^{c} \sigma_{r r^{\prime}} \cos \phi_{r r^{\prime}}-\sum_{r r^{\prime}} J_{r r^{\prime}}^{2 c} \cos \left(2 \phi_{r r^{\prime}}\right) \\
& -\sum_{r r^{\prime}} J_{r r^{\prime}}^{s} \sigma_{r r^{\prime}} \mathbf{n}_{r} \mathbf{n}_{r^{\prime}}-\sum_{r r^{\prime}} J_{r r^{\prime}}^{2 s} Q_{r}^{a b} Q_{r^{\prime}}^{a b}
\end{aligned}
$$

Here the summation goes over sites $r=(i, \tau)$ in the space-time lattice, $\phi_{r}$ is the phase variable conjugate to $N_{r}$, $\phi_{r r^{\prime}}=\phi_{r}-\phi_{r^{\prime}}, Q_{r}^{a b}=n_{r}^{a} n_{r}^{b}-\delta^{a b} / 3$ is a nematic order parameter, and $\sigma_{r r^{\prime}}= \pm 1$ is an Ising field that lives on the links rather than the sites. The coupling constants are $J_{r, r \pm \hat{\tau}}^{c}=(\epsilon u)^{-1}, J_{r, r \pm \hat{\tau}}^{s}=(\epsilon g)^{-1}, J_{r, r \pm\{\hat{x}, \hat{y}, \hat{z}\}}^{c, s}=\epsilon J|\chi|$, $J_{r, r \pm \hat{\tau}}^{2 c}=J_{r, r \pm \hat{\tau}}^{2 s}=0, J_{r, r \pm\{\hat{x}, \hat{y}, \hat{z}\}}^{2 c, s}=-\epsilon J / 4$, with $\chi$ being the saddle point value of the Hubbard-Stratanovich field. The usual periodic boundary conditions for $\phi$, $\mathbf{n}$, and $\sigma$ are assumed at $\tau=0$ and $\tau=\beta$. In writing Eq. (2) we omitted the Berry phase terms that are important for quantitative calculations of the phase diagram but not for the symmetry arguments discussed below.

Equation (2) has a $Z_{2}$ gauge symmetry $\phi_{r} \rightarrow \phi_{r}+$ $\frac{\pi}{2}\left(1-\boldsymbol{\epsilon}_{r}\right), \mathbf{n}_{r} \rightarrow \boldsymbol{\epsilon}_{r} \mathbf{n}_{r}, \sigma_{r, r+\hat{a}} \rightarrow \boldsymbol{\epsilon}_{r} \sigma_{r, r+\hat{a}} \boldsymbol{\epsilon}_{r+\hat{a}}$, where $\epsilon_{r}= \pm 1$. Such a symmetry was pointed out earlier in $[10,29]$, where it was observed that the physical order parameter is a complex vector $\mathbf{d}=e^{i \phi} \mathbf{n}$ (not $\phi$ and $\mathbf{n}$ separately) that has a symmetry of $e^{i \phi} \rightarrow-e^{i \phi}, \mathbf{n} \rightarrow-\mathbf{n}$. Equation (2) is the simplest action consistent with the charge $\mathrm{U}(1)$, spin $\mathrm{SO}(3)$, and gauge $Z_{2}$ symmetries of the model. Another term allowed by the $Z_{2}$ symmetry is the analog of the Maxwell terms for the lattice gauge models $S_{\sigma}=-K \sum_{\square} \prod_{\square} \sigma_{i j}$, where the summation goes over plaquettes in a $(d+1)$-dimensional lattice. This term may be generated by integrating out the high energy degrees of freedom.

The existence of the local symmetry imposes important constraints on the possible order parameters and symmetry breaking states of the system. Only order parameters that are gauge invariant may acquire expectation values. For example, the expectation values $\langle\mathbf{n}\rangle \neq 0$ or $\left\langle e^{i \phi}\right\rangle \neq 0$ are not allowed in the models described by Eq. (2), since $\mathbf{n}_{i}$ and $e^{i \phi_{i}}$ are not invariant under $Z_{2}$ gauge transformations. Physically this restriction arises from the fact that the wave function $\Psi(\mathbf{n})$ should be an even or odd function of $\mathbf{n}$ for $N$ even or odd, respectively. Below we consider several examples of the order parameters that are gauge invariant and review the broken symmetry phases that they lead to (see Fig. 1). Polar BEC phase (PC) has $\langle\mathbf{d}\rangle=\left\langle e^{i \phi} \mathbf{n}\right\rangle \neq 0$. It breaks both charge and spin symmetries and is the phase where atoms are condensed directly. The nematic insulator phase has $\left\langle Q_{a b}\right\rangle=\left\langle n_{a} n_{b}-\delta_{a b} / 3\right\rangle \neq 0$ and breaks only the spin $\mathrm{SO}(3)$ symmetry. Spin singlet condensate (SSC) $\left\langle e^{i 2 \phi}\right\rangle \neq 0$ breaks the $\mathrm{U}(1)$ symmetry but not $\mathrm{SO}(3)$. It corresponds to a condensate of singlet pairs of atoms. Another possible phase is a SCP in phase in which both $\left\langle e^{i 2 \phi}\right\rangle \neq 0$ and $\left\langle Q_{a b}\right\rangle \neq 0$ but $\langle\mathbf{d}\rangle=0$. This phase breaks both $\mathrm{U}(1)$ and $\mathrm{SO}(3)$ symmetries, however, it is fundamen- tally different from the polar condensate phase [30,31]. Finally, SSI is a phase where no field has an expectation value, so neither $\mathrm{U}(1)$ nor $\mathrm{SO}(3)$ symmetries are broken. We note that for a finite number of traps, when spontaneous symmetry breaking is not possible, one can observe signatures of the transitions by measuring fluctuations in the number of atoms, atom pairs, and spins [20].

It is interesting to point out that phases NI, SSC, and SCP correspond to the fragmented BEC discussed by Nozieres and Saint James. The part emphasized in [22] is that the fragmented state has no macroscopic population of the $k=0$ state for the bosons. Another interesting aspect of these states is that they achieve fractionalization of topological objects. The unfragmented PC phase has topological excitations that are composites of a $1 / 2$ vortex in the charge sector and $\pi$ disclination in the spin sector. Here the $1 / 2$ charge vortex is a topological defect around which the phase $\phi$ winds by $\pi$, and $e^{i \phi}$ changes sign. $\pi$ disclination in the spin sector is introduced as a topological defect around which $\mathbf{n}$ changes sign (vortex like objects in $\mathbf{n}$ are called merons, so $\pi$ disclination is one-half of a meron). Both $1 / 2$ vortex and $\pi$ disclination are pointlike objects in two dimensions and lines in three dimensions. No individual $1 / 2$ charge vortices or $\pi$ disclinations are allowed in the PC phase. The spin and charge parts of the vortex are always glued together. A condensate of singlet pairs only, the SSC phase, has $1 / 2$ charge vortices as separate excitations, but no spin vortices; the nematic phase NI has $\pi$ disclinations and no charge vortices. A strong coupling pairing phase will have $1 / 2$ charge vortices and $\pi$ disclinations as separate excitations.

Establishing the nature of the broken symmetry does not fully characterize models with gauge symmetry. Another important aspect of the system described by Eq. (2) is the possibility of confining and deconfining phases of the $Z_{2}$ gauge theory in dimension $d+1 \geq 3$. The difference between the confining and deconfining phases relies on the concept of topological order [23-26] and has important implications on the nature of excitations in the system. Topological order may coexist with any true long range order, so any of the phases reviewed earlier may be confining or deconfining. For the pure $Z_{2}$ gauge models the confining and deconfining phases may be distinguished using Wilson loops, which are characterized by an area law for the confining phase and by a perimeter law for the deconfining phase. With matter field present, Wilson loops may no longer be used to discriminate between the two phases [23]; however, distinction between them survives, and there is a phase transition between the two. A simple way to understand the transition is to think of it as condensation of visons, topological excitations of the $Z_{2}$ gauge theory that describe frustrated plaquettes $\prod_{\square} \sigma_{r r^{\prime}}=-1$. Particles that carry a $Z_{2}$ charge are frustrated when traveling around a vison, so when visons are condensed such particles may not propagate coherently and we have a confining phase. When visons are 
not condensed, they are finite energy topological excitations and we have a deconfined phase. The presence of finite energy visons leads to an additional degeneracy of the gauge models in a deconfined state on manifolds with non-trivial degeneracy. Systems with $Z_{2}$ gauge symmetries in condensed matter have already been discussed by Lammert et al. [32] in connection with liquid crystals and Sachdev and co-workers [24] and Senthil and Fisher [26] in connection with high temperature superconductors. For our system the discussion above implies that all the broken symmetry states, as well as a spin singlet insulating state, where neither $\mathrm{U}(1)$ nor $\mathrm{SO}(3)$ symmetries are broken, come in two varieties: confining and deconfining. Detailed comparison between confining and deconfining versions of various phases will be given in [28]. Here we note only that some of the most striking implications of deconfinement appear for the SSI phase. The deconfining SSI $^{*}$ phase allows charge $b=e^{i \phi}$, and spin $\mathbf{n}$ carrying excitations propagate independently, which changes dramatically the spectrum of low energy excitations as discussed in [33].

To summarize, we reviewed several novel phenomena that take place for $F=1$ Bose gases with antiferromagnetic interactions. We showed that in optical lattices they will have several distinct superfluid and Mott insulator phases depending on the density of atoms, the scattering lengths, and the strength of the periodic potential. Another possibility suggested in this paper is a class of fractionalized phases of spinor BEC, characterized by the topological order that may coexist with any true long range order in the system. Quantitative analysis of experimental systems will be given in subsequent publications [28]. Discussion in this paper may be generalized to systems with higher hyperfine spins. Ideas discussed in this paper for spinor condensates are also relevant to several condensed matter systems, including triplet superconductors [29], multicomponent quantum Hall effects [30], and incommensurate spin density waves [33].

We thank M.P. A. Fisher, F. D. Haldane, H. Y. Kee, Y. B. Kim, M. Lukin, C. Nayak, S. Sachdev, J. Sethna, T. Senthil, H. T. Stoof, A. Vishwanath, and P. Wiegnman for useful discussions. This work was partially supported by NSF Grant No. DMR-0132874.

*Permanent address: Institute for Theoretical Physics, University of Utrecht, Princetonplein 5, 3584 CC Utrecht, The Netherlands
[1] D. M. Stamper-Kurn et al., Phys. Rev. Lett. 80, 2027 (1998).

[2] J. Stenger et al., Nature (London) 396, 345 (1998).

[3] W. Ketterle, cond-mat/0005001.

[4] A. Sorensen et al., Nature (London) 409, 63 (2001).

[5] T. L. Ho, Phys. Rev. Lett. 81, 742 (1998).

[6] T. Ohmi and K. Machida, J. Phys. Soc. Jpn. 67, 1822 (1998).

[7] C. K. Law, H. Pu, and N. P. Bigelow, Phys. Rev. Lett. 81, 5257 (1998).

[8] T. L. Ho and S. K. Yip, Phys. Rev. Lett. 84, 4031 (2000).

[9] Y. Castin and C. Herzog, cond-mat/0012040.

[10] F. Zhou, Phys. Rev. Lett. 87, 080401 (2001); F. Zhou and F. D. Haldane, Report No. ITP-UU-00/51(2000).

[11] G. Grynberg et al., Phys. Rev. Lett. 70, 2249 (1993).

[12] S. E. Hamann et al., Phys. Rev. Lett. 80, 4149 (1998).

[13] S. Friebel et al., Phys. Rev. A 57, R20 (1998).

[14] M. Rayzen, C. Salomon, and Q. Niu, Phys. Today 50, No. 7, 30 (1997).

[15] L. Guidoni and P. Verkerk, Phys. Rev. Lett. 79, 3363 (1997).

[16] K. I. Petsas, A. B. Coates, and G. Grynberg, Phys. Rev. A 50, 5173 (1994).

[17] I. H. Deutsch and P.S. Jessen, Phys. Rev. A 57, 1972 (1998).

[18] D. J. Han et al., Phys. Rev. Lett. 85, 724 (2000).

[19] D. Jaksch et al., Phys. Rev. Lett. 81, 3108 (1998).

[20] B. P. Anderson and M. Kasevich, Science 282, 1686 (1998); C. Orzel, A. Tuchman, M. Fenselau, M. Yasuda, and M. Kasevich, Science 291, 2386 (2001).

[21] M. Greiner et al., Nature (London) 415, 39 (2002).

[22] P. Nozieres and D. Saint James, J. Phys. (Paris) 43, 1133 (1982).

[23] J. B. Kogut, Rev. Mod. Phys. 51, 659 (1979); E. Fradkin and S. Shenker, Phys. Rev. D 19, 3682 (1979).

[24] N. Read and S. Sachdev, Phys. Rev. Lett. 66, 1773 (1991); R. Jalabert and S. Sachdev, Phys. Rev. B 44, 686 (1991).

[25] X. G. Wen and Q. Niu, Phys. Rev. B 41, 9377 (1990).

[26] T. Senthil and M. P. A. Fisher, cond-mat/9910224.

[27] S. Chakravarty and S. Kivelson, cond-mat/0012305.

[28] E. Demler, F. Zhou, and D. Haldane, Report No. ITP-UU01/09(2001)

[29] Eugene Demler, Chetan Nayak, Hae-Young Kee, Yong Baek Kim, and T. Senthil, cond-mat/0105446.

[30] E. Demler, C. Nayak, and S. Das Sarma, Phys. Rev. Lett. 86, 1853 (2001).

[31] Y. B. Kim et al., cond-mat/0011459.

[32] P. E. Lammert, D. S. Rokhsar, and J. Toner, Phys. Rev. Lett. 70, 1650 (1993).

[33] Ying Zhang, Eugene Demler, and Subir Sachdev, condmat/0112343. 